\section{$\mathrm{T}_{\text {reg }}$ cell division of labor}

The relative contributions of inducible regulatory $\mathrm{T}$ cells ( $\mathrm{iT}_{\text {reg }}$ cells) and thymus-derived regulatory $\mathrm{T}$ cells ( $\mathrm{nT}_{\text {reg }}$ cells) to various aspects of immune homeostasis remain unclear. In Nature, Rudensky and colleagues show that deficiency in $\mathrm{iT}_{\text {reg }}$ cells does not lead to systemic or tissue-specific autoimmune pathology or enhanced proinflammatory responses of helper $T$ cells of the $T_{H} 1$ or $T_{H} 17$ subset but leads to spontaneous $T$ helper type $2\left(\mathrm{~T}_{\mathrm{H}} 2\right)$ pathologies at mucosal sites. The authors use mice deficient in the transforming growth factor- $\beta$ (TGF$\beta$ ) enhancer CNS1, which cannot induce expression of the transcription factor Foxp3 dependent on TGF- $\beta$, and as a consequence have no $\mathrm{iT}_{\text {reg }}$ cells, although the number of $\mathrm{nT}_{\text {reg }}$ cells is normal. CNS1-deficient mice show dysregulated $\mathrm{T}_{\mathrm{H}} 2$ responses that lead to spontaneous inflammation of the airway and gastrointestinal tract. Loss of $\mathrm{T}_{\mathrm{H}} 2$ homeostasis leads to perturbations in the composition of the microbial community in the gut. These results suggest that $\mathrm{nT}_{\text {reg }}$ and $\mathrm{iT}_{\text {reg }}$ cells not only have distinct mechanistic requirements for differentiation but also specific functions in immune homeostasis.

IV Nature (8 February 2012) doi:10.1038/nature10772

\section{Trefoils and type 2 immunity}

Type 2 immunity is elicited during parasitic infection and contributes to the immunopathology of allergic diseases. Initiators of type 2 responses have attracted considerable debate. In the Journal of Experimental Medicine, Herbert and colleagues show that trefoil factor 2 (TFF2) is an early initiator of type 2 immunity in response to Nippostrongylus brasiliensis. Lung epithelial damage triggers release of TFF2. Asthmatic children have higher expression of TFF2. TFF2 signaling through the receptor CXCR4 leads to elaboration of the type 2-inducing cytokine interleukin 33 (IL-33); this promotes expression of IL-4, IL-13 and RELM- $\beta$, which are necessary for worm expulsion. TFF2-deficient mice have greater worm burdens and delayed type 2 immune responsiveness. These findings suggest that TFF 2 contributes to the early type 2 immune response to parasites and aberrantly to asthma.

$L A D$

J. Exp. Med. (13 February 2012) doi:10.1084/jem.20110079

\section{Lin28 confers fetal lymphopoiesis}

Early hematopoiesis occurs in the fetal liver but transitions to the bone marrow at later stages of development. Distinct innatelike lymphocytes arise from the fetal liver progenitors, but what distinguishes fetal cells from adult cells has remained unknown. In Science, Muljo and colleagues show that differences in the expression of Lin28 microRNA regulate fetal hematopoiesis in both mice and humans. Lin28 suppresses the Let-7 family of microRNA, present in abundance in adult hematopoietic progenitor cells. Ectopic expression of Lin28 transforms adult bone-marrow-cell potential to that arising from fetal liver-derived progenitors. These include more production of innate-like B-1a cells and marginal-zone B cells, certain $\gamma \delta$ T cells and PLZF ${ }^{+}$ invariant natural killer cells. These findings provide a mechanistic basis for the distinct waves of lymphocyte development that occur during ontogeny.

Science (16 February 2012) 10.1126/science.1216557

\section{LAD}

\section{Turning on plant immunity}

Infection of plants triggers a switch of resources from growth to defense characterized by considerable transcriptional reprogramming involving thousands of genes. In Current Biology, Dong and colleagues use Arabidopsis to identify the transcription factor TBF1 as a key component of this switch from growth to defense. Infection results in the recognition of microbe-associated molecular patterns, which leads to effector-triggered immunity. In addition, infection triggers a longer-lived systemic response mediated by salicylic acid. Both these defense pathways result in the derepression of TBF1, which allows it to directly regulate nearly 3,000 genes. Among the effects is repression of growth-related genes, such as those encoding molecules involved in chloroplast function, as well as the positive regulation of immune-related genes. Infection normally results in less growth as resources are shifted to defense; however, this effect is alleviated in plants with mutant TBF1. TBF1 is therefore involved in sensing the metabolic changes associated with infection and in placing the host plant onto a 'war footing'.

Curr. Biol. (24 January 2012) doi:10.1016/j.cub.2011.12.015

\section{Cytotoxic pDCs}

Imiquimod is an agonist of Toll-like receptor 7 (TLR7) and TLR8 and is used clinically for the treatment of certain forms of skin cancer. In the Journal of Clinical Investigation, Sibilia and colleagues demonstrate that imiquimod can trigger tumor clearance through the stimulation of 'killer' plasmacytoid dendritic cells ( $p D C s)$. Short-term application of imiquimod results in dermal cell death but not in any obvious production of inflammatory cytokines in the skin. However, imiquimod triggers skin mast cells to release the chemokine CCL2, which recruits pDCs. Imiquimod mediates protection against tumor challenge and, unexpectedly, also works in the complete absence of any adaptive immunity or natural killer cells. Instead, imiquimod activates pDCs to express soluble cytotoxic factors such as TRAIL and granzyme B, which could directly kill tumor cells. The production and signaling of interferon- $\alpha$ in response to imiquimod is essential for the upregulation of pDC cytotoxic factors. Thus, pDCs are important in the direct elimination of tumors.

ZF

\title{
Advanced T cell profiling
}

Various combinations of techniques have been used to functionally characterize lymphoid populations. In Immunity, Davis and colleagues use the single-cell mass spectrometry technique CyTOF to provide a comprehensive picture of $\mathrm{CD} 8^{+} \mathrm{T}$ cell phenotypic and functional diversity. Using heavy metal isotope-labeled antibodies and tetramers of peptide and major histocompatibility complex, the authors survey various functional attributes, such as intracellular cytokines, degranulation, cytotoxic granule components and $\mathrm{T}$ cell antigen receptor specificity, to examine the relationship between surface-marker phenotypes and the function of $\mathrm{T}$ cells in the context of their specificity for epitopes of influenza virus, cytomegalovirus and Epstein-Barr virus. They use principal-component analysis to simultaneously analyze and visualize such a large data set. Their analysis suggests that $\mathrm{CD} 8^{+} \mathrm{T}$ cells have a greater degree of functional and phenotypic complexity than previously appreciated, along a continuum of differentiation and maturation stages that can vary depending on the antigen specificity of the cells. $I V$ Immunity 36, 142-152 (2012) 